\title{
Multiplier ideal sheaves and the Kähler-Ricci flow
}

\author{
D.H. Phong ${ }^{1}$, Natasa Sesum ${ }^{1}$ And Jacob Sturm ${ }^{2}$
}

\begin{abstract}
Multiplier ideal sheaves are constructed as obstructions to the convergence of the Kähler-Ricci flow on Fano manifolds, following earlier constructions of Kohn, Siu, and Nadel, and using the recent estimates of Kolodziej and Perelman.
\end{abstract}

\section{Introduction}

The global obstruction to the existence of a Hermitian-Einstein metric on a holomorphic vector bundle is well known to be encoded in a destabilizing sheaf, thanks to the works of Donaldson [9, 10] and Uhlenbeck-Yau [27]. It is expected that this should also be the case for general canonical metrics in Kähler geometry. For Kähler-Einstein metrics on Fano manifolds, obstructing sheaves have been constructed by Nadel [15] as multiplier ideal sheaves, following ideas of Kohn [11] and Siu [23]. This formulation in terms of multiplier ideal sheaves opens up many possibilities for relations with complex and algebraic geometry [7, 19, 24, 28].

The obstructing multiplier ideal sheaves are not expected to be unique. Nadel's construction is based on the method of continuity for solving a specific Monge-Ampère equation for Kähler-Einstein metrics. It has always been desirable to construct also an obstructing multiplier ideal sheaf from the Kähler-Ricci flow. The purpose of this note is to show that this can be easily done, using the recent estimates of Kolodziej [12,13] and Perelman [18]. In effect, Kolodziej's estimates provide a Harnack estimate for the Monge-Ampère equation, which is elliptic, and Perelman's estimate reduces the Kähler-Ricci flow, which is parabolic, to the Monge-Ampère equation. Similar ideas were exploited by Tian-Zhu [26] in their proof of an inequality of Harnack type for the Kähler-Ricci flow.

\section{The multiplier ideal sheaf}

Let $X$ be an $n$-dimensional compact Kähler manifold, equipped with a Kähler form $\omega_{0}$ with $\mu \omega_{0} \in c_{1}(X)$, where $\mu$ is a constant. The Kähler-Ricci 
flow is the flow defined by

$$
\dot{g}_{\bar{k} j}=-\left(R_{\bar{k} j}-\mu g_{\bar{k} j}\right)
$$

where $g_{\bar{k} j}=g_{\bar{k} j}(t)$ is a metric evolving in time $t$ with initial value $g_{\bar{k} j}(0)=$ $\hat{g}_{\bar{k} j}$, and $R_{\bar{k} j}=-\partial_{j} \partial_{\bar{k}} \log \operatorname{det} g_{\bar{q} p}$ is its Ricci curvature. Since the KählerRicci flow preserves the Kähler class of the metric, we may set $g_{\bar{k} j}=\hat{g}_{\bar{k} j}+$ $\partial_{j} \partial_{\bar{k}} \phi$, and the Kähler-Ricci flow can be reformulated as

$$
\dot{\phi}=\log \frac{\omega_{\phi}^{n}}{\omega_{0}^{n}}+\mu \phi-\hat{f}, \quad \phi(0)=c_{0},
$$

where we have set $\omega_{\phi}=\frac{i}{2} g_{\bar{k} j} d z^{j} \wedge d \bar{z}^{k}$, and $\hat{f}$ is the Ricci potential for the metric $\hat{g}_{\bar{k} j}$, that is, the $C^{\infty}$ function defined by the equation $\hat{R}_{\bar{k} j}-\mu \hat{g}_{\bar{k} j}=$ $\partial_{j} \partial_{\bar{k}} \hat{f}$, normalized by the condition that

$$
\int_{X} \mathrm{e}^{\hat{f}} \omega_{0}^{n}=\int_{X} \omega_{0}^{n} \equiv V
$$

Here and henceforth, $\hat{R}_{\bar{k} j}$ denotes the Ricci curvature of $\hat{g}_{\bar{k} j}$, with similar conventions for all the other curvatures of $\hat{g}_{\bar{k} j}$. The initial potential $c_{0}$ is a constant, so that the initial metric coincides with $\hat{g}_{\bar{k} j}$. The Kähler-Ricci flow exists for all time $t>0$ [3], and the main issue is its convergence. Henceforth, we shall restrict to the case $c_{1}(X)>0$ of Fano manifolds unless indicated explicitly otherwise, and set $\mu=1$.

Theorem 2.1. Let $X$ be an $n$-dimensional compact Kähler manifold with $c_{1}(X)>0$.

(i) Consider the Kähler-Ricci flow (2.2) for potentials $\phi$, with the initial value $c_{0}$ specified by (2.10) below. If there exists some $p>1$ with

$$
\sup _{t \geq 0} \int_{X} \mathrm{e}^{-p \phi} \omega_{0}^{n}<\infty
$$

then there exists a sequence of times $t_{i} \rightarrow+\infty$ with $g_{\bar{k} j}\left(t_{i}\right)$ converging in $C^{\infty}$ to a Kähler-Einstein metric. If in addition $X$ admits no nontrivial holomorphic vector field, then the whole flows (2.1) and (2.2) converge in $C^{\infty}$.

(ii) If $X$ does not admit a Kähler-Einstein metric, then for each $p>1$, there exists a function $\psi$ which is a $L^{1}$ limit point of $\phi-\frac{1}{V} \int_{X} \phi \omega_{0}^{n}$, 
with the following property. Let the multiplier ideal sheaf $\mathcal{J}(p \psi)$ be the sheaf with stalk at $z$ defined by

$$
\mathcal{J}_{z}(p \psi)=\left\{f ; \quad \exists U \ni z, \quad f \in \mathcal{O}(U), \quad \int_{U}|f|^{2} \mathrm{e}^{-p \psi} \omega_{0}^{n}<\infty\right\},
$$

where $U \subset X$ is open, and $\mathcal{O}(U)$ denotes the space of holomorphic functions on $U$. Then $\mathcal{J}(p \psi)$ defines a proper coherent analytic sheaf on $X$, with acyclic cohomology, i.e.,

$$
H^{q}\left(X, K_{X}^{-[p]} \otimes \mathcal{J}(p \psi)\right)=0, \quad q \geq 1
$$

If $X$ admits a compact group $G$ of holomorphic automorphisms, and $\hat{g}_{\bar{k} j}$ is $G$-invariant, then $\mathcal{J}(p \psi)$ and the corresponding subscheme are also $G$-invariant.

In Part (i), once the convergence of a subsequence $g_{\bar{k} j}\left(t_{i}\right)$ has been established and $X$ is known to admit a Kähler-Einstein metric, it follows from an unpublished result of Perelman that the full Kähler-Ricci flow must then converge. An extension of Perelman's result to Kähler-Ricci solitons is given in Tian-Zhu [26]. For the sake of completeness, we have provided a short self-contained proof of the Kähler-Einstein case, in our context and under the simplifying assumption of no non-trivial holomorphic vector fields.

Part (ii) is of course exactly the same as in the method of continuity for the Monge-Ampère equation used by Nadel [15], in the formulation of Demailly-Kollár [7]. We divide the proof of Theorem 2.1 into several lemmas.

First, we need to recall the fundamental recent result of Perelman. Let the Kähler-Ricci flow be defined by (2.1), and for each time $t$, define the Ricci potential $f$ by

$$
R_{\bar{k} j}-g_{\bar{k} j}=\partial_{j} \partial_{\bar{k}} f, \quad \frac{1}{V} \int_{X} \mathrm{e}^{f} \omega_{\phi}^{n}=1 .
$$

(In particular, at time $t=0$, the Ricci potential coincides with the function $\hat{f}$ defined earlier.) Then Perelman [18] (see also [22]) has shown that

$$
\sup _{t \geq 0}\left(\|f\|_{C^{0}}+\|\nabla f\|_{C^{0}}+\|\Delta f\|_{C^{0}}\right)<+\infty
$$

with Laplacians and norms taken with respect to the metric $g_{\bar{k} j}$.

Next, we specify the value of the initial potential $c_{0}$ in $(2.2)$, following Chen and Tian [5] (see also [14]). The underlying observation is that 
$\|\nabla \dot{\phi}\|_{L^{2}}^{2}$, and hence the integral

$$
\int_{0}^{\infty} \mathrm{e}^{-t}\|\nabla \dot{\phi}\|_{L^{2}}^{2} d t
$$

does not depend on the choice of initial value $c_{0}$ for the flow (2.2). Indeed, given a flow $\phi$ with initial value $c_{0}$, the function $\tilde{\phi}=\phi+\left(\tilde{c}_{0}-c_{0}\right) \mathrm{e}^{t}$ satisfies the same flow with initial value $\tilde{c}_{0}$, and hence, by uniqueness, must coincide with the flow with initial value $\tilde{c}_{0}$. Clearly, $\nabla \phi=\nabla \tilde{\phi}$, hence the assertion. Note also that the integral in (2.9) is always finite, in view of Perelman's estimate (2.8). Following [5], we use this common value to choose the initial value $c_{0}$ in $(2.2)$,

$$
c_{0}=\int_{0}^{\infty} \mathrm{e}^{-t}\|\nabla \dot{\phi}\|_{L^{2}}^{2} d t+\frac{1}{V} \int_{X} \hat{f} \omega_{0}^{n} .
$$

A specific choice of initial data is clearly necessary to discuss the convergence of the Kähler-Ricci flow (2.2) for potentials, in view of the fact that different initial data for $\phi$ lead to flows differing by terms blowing up in time. We will see below that the choice (2.10) is the right choice.

The first indication is that, with the choice (2.10) for the initial data (2.2), Perelman's estimate for $h$ implies

$$
\sup _{t \geq 0}\|\dot{\phi}\|_{C^{0}}<\infty \text {. }
$$

To see this, we note that $f+\dot{\phi}$ is a constant, since $\partial \bar{\partial}(f+\dot{\phi})=0$. It suffices to show then that the average $\alpha(t) \equiv 1 / V \int_{X} \dot{\phi} \omega_{\phi}^{n}$ of $\dot{\phi}$ is uniformly bounded in absolute value, since $|f|$ already is, by Perelman's estimate (2.8). Now differentiating the equation (2.2) gives $\partial_{t} \dot{\phi}=\Delta \dot{\phi}+\dot{\phi}$, and hence

$$
\begin{aligned}
\partial_{t}\left(\frac{1}{V} \int_{X} \dot{\phi} \omega_{\phi}^{n}\right) & =\frac{1}{V} \int_{X}(\Delta \dot{\phi}+\dot{\phi}) \omega_{\phi}^{n}+\frac{1}{V} \int_{X} \dot{\phi} \Delta \dot{\phi} \omega_{\phi}^{n} \\
& =\frac{1}{V} \int_{X} \dot{\phi} \omega_{\phi}^{n}-\|\nabla \dot{\phi}\|_{L^{2}}^{2} .
\end{aligned}
$$

This is a differential equation for $\alpha(t)$ which can be integrated, giving

$$
\begin{aligned}
\mathrm{e}^{-t} \alpha(t) & =\alpha(0)-\int_{0}^{t} \mathrm{e}^{-s}\|\nabla \dot{\phi}(s)\|_{L^{2}}^{2} d s \\
& =\int_{t}^{\infty} \mathrm{e}^{-s}\|\nabla \dot{\phi}(s)\|_{L^{2}}^{2} d s
\end{aligned}
$$


in view of (2.10) and the fact that at time $t=0$, we have $\dot{\phi}=c_{0}-\hat{f}$. It follows that

$$
\begin{aligned}
0 \leq \alpha(t) & =\int_{t}^{\infty} \mathrm{e}^{-(s-t)}\|\nabla \dot{\phi}(s)\|_{L^{2}}^{2} d s \\
& \leq C \int_{t}^{\infty} \mathrm{e}^{-(s-t)} d s \leq C
\end{aligned}
$$

where we have applied Perelman's uniform bound for $\|\nabla \dot{\phi}\|_{C^{0}}$. This proves (2.11).

More systematically, uniform bounds for $\phi$ and $g_{\bar{k} j}$ are now equivalent.

Lemma 2.2. Let $X$ be a compact Kähler manifold, with Kähler form $\omega_{0} \in$ $c_{1}(X)$, and consider the Kähler-Ricci flows (2.1) and (2.2) for $g_{\bar{k} j}$ and $\phi$, respectively. Let the initial value $c_{0}$ for $\phi$ be given by (2.10). Then $\|\phi\|_{C^{m}}$ is uniformly bounded for all $m$ if and only if $\left\|g_{\bar{k}_{j}}\right\|_{C^{m}}$ is bounded for all $m$ (here norms are taken with respect to a fixed reference metric, say $\hat{g}_{\bar{k} j}$ ). The flow for $g_{\bar{k} j}$ converges in $C^{\infty}$ if and only if the flow for $\phi$ converges in $C^{\infty}$.

Proof. Clearly, the convergence/boundedness of the potentials $\phi$ 's implies the convergence/boundedness of the metrics $g_{\bar{k} j}$. Conversely, the convergence/boundedness of the metrics implies the convergence/boundedness of $\partial \bar{\partial} \phi$, so it suffices to establish the convergence/boundedness of the averages of $\phi$ with respect to the volume forms $\omega_{\phi}^{n}$. Since the flow implies

$$
\frac{1}{V} \int_{X} \phi \omega_{\phi}^{n}=\frac{1}{V} \int_{X} \dot{\phi} \omega_{\phi}^{n}-\frac{1}{V} \int_{X} \log \frac{\omega_{\phi}^{n}}{\omega_{0}^{n}} \omega_{\phi}^{n}+\frac{1}{V} \int_{X} \hat{f} \omega_{\phi}^{n}
$$

and $|\alpha(t)|$ is bounded in view of (2.14), it follows that $\left|1 / V \int_{X} \phi \omega_{\phi}^{n}\right|$ is bounded in either case. Assume now that $g_{\bar{k} j}$ converges. We wish to show the convergence of $1 / V \int_{X} \phi \omega_{\phi}^{n}$, and thus of $\alpha(t)$.

The convergence of $g_{\bar{k} j}$ implies that $X$ admits a Kähler-Einstein metric. By a theorem of Bando-Mabuchi [2], the Mabuchi $K$-energy functional must be then bounded from below. This is well known to imply in turn that $\|\nabla \dot{\phi}\|_{L^{2}} \rightarrow 0$ as $t \rightarrow+\infty$ (see e.g., [21] equation (2.10) and subsequent paragraph). But with the choice (2.10) for initial data for $(2.2)$, we have the estimate (2.14), which implies now that $\alpha(t) \rightarrow 0$.

Lemma 2.3. Let $X$ be a compact Kähler manifold, and consider the Kähler-Ricci flow as defined by (2.1) and (2.2) with $\omega_{0} \in c_{1}(X)$, and the 
initial value $c_{0}$ for $\phi$ specified by (2.10). Then for any $p>1$, we have

$$
\sup _{t \geq 0} \int_{X} \mathrm{e}^{-p \phi} \omega_{0}^{n}<\infty \Longleftrightarrow \sup _{t \geq 0}\|\phi\|_{C^{0}}<\infty
$$

Proof. This lemma is a direct consequence of the above results of Perelman combined with results of Kolodziej. Clearly, the uniform boundedness of the $C^{0}$ norm of $\phi$ implies the uniform boundedness of $\left\|\mathrm{e}^{-\phi}\right\|_{L^{p}(X)}$. To show the converse, we consider the following Monge-Ampère equation

$$
\operatorname{det}\left(\hat{g}_{\bar{k} j}+\partial_{j} \partial_{\bar{k}} \phi\right)=\Phi \operatorname{det} \hat{g}_{\bar{k} j}
$$

where $\Phi$ is a smooth strictly positive function. Then Kolodziej $[12,13]$ has shown that, for any $p>1$, the solution $\phi$ must satisfy the a priori bound

$$
\operatorname{osc}_{X} \phi \equiv \sup _{X} \phi-\inf _{X} \phi \leq C_{p}
$$

for some constant $C_{p}$ which is bounded if $\|\Phi\|_{L^{p}(X)}$ is bounded. Now the Kähler-Ricci flow (2.2) can be rewritten in the form (2.17) with $\Phi=\exp (\hat{f}-$ $\phi+\dot{\phi})$. By Perelman's estimate (2.11), $\|\Phi\|_{L^{p}(X)}$ is uniformly bounded if and only if $\left\|\mathrm{e}^{-\phi}\right\|_{L^{p}(X)}$ is uniformly bounded. Combined with Kolodziej's result, we see that the uniform boundedness of $\left\|\mathrm{e}^{-\phi}\right\|_{L^{p}(X)}$ implies the uniform boundedness of $\operatorname{osc}_{X} \phi$.

To obtain a bound for $\|\phi\|_{C^{0}}$ from osc $\phi$, it suffices to produce a lower bound for $\sup _{X} \phi$ and an upper bound for $\inf _{X} \phi$. Now, from Perelman's estimate, we have

$$
C_{1} \mathrm{e}^{\hat{f}-\phi+\dot{\phi}} \omega_{0}^{n} \leq \mathrm{e}^{-\phi} \omega_{0}^{n} \leq C_{2} \mathrm{e}^{\hat{f}-\phi+\dot{\phi}} \omega_{0}^{n}
$$

and hence, integrating and recalling that $\mathrm{e}^{\hat{f}-\phi+\dot{\phi}} \omega_{0}^{n}=\omega_{\phi}^{n}$ has the same volume as $\omega_{0}^{n}$,

$$
C_{1} \leq \frac{1}{V} \int_{X} \mathrm{e}^{-\phi} \omega_{0}^{n} \leq C_{2} .
$$

This implies at once that

$$
\sup _{X} \phi \geq-\log C_{2}, \quad \inf _{X} \phi \leq-\log C_{1}
$$

The proof is complete. 
Lemma 2.4. Let $X$ be a compact Kähler manifold with Kähler form $\omega_{0}$ satifying $\mu \omega_{0} \in c_{1}(X)$, where $\mu$ is any constant. Let the Kähler-Ricci flow be defined by (2.2). Then we have the a priori estimates

$$
\begin{aligned}
\sup _{t \geq 0}\|\phi\|_{C^{0}} & \leq A_{0}<\infty \Longleftrightarrow \sup _{t \geq 0}\|\phi\|_{C^{k}} \\
& \leq A_{k}<\infty, \quad \forall k \in \mathbf{N} .
\end{aligned}
$$

Proof. This is the parabolic analogue of Yau's and Aubin's well-known result $[1,29]$, namely, that the same statement holds for the solution $\phi$ of the elliptic Monge-Ampère equation (2.17), with the corresponding constants $A_{k}$ depending on the $C^{\infty}$ norms of the right hand side $\Phi$. Now the KählerRicci flow can be rewritten in the form (2.17), with $\Phi=\exp (\hat{f}-\phi+\dot{\phi})$. The hypothesis $\|\phi\|_{C^{0}} \leq A_{0}$ implies control of $\|\Phi\|_{C^{0}}$, in view of Perelman's estimate. However, we do not have control of all the $C^{\infty}$ norms of $\Phi$, and hence Yau's a priori estimates cannot be quoted directly.

Thus we have to go through a full parabolic analogue of Yau's arguments, and make sure that it goes through without any estimate on $\dot{\phi}$ which is not provided by Perelman's result. The arguments here are completely parallel to Yau's, but we take this opportunity to present a more streamlined version. The parabolic analogues of several key identities are also made more explicit. They turn out to be quite simple, and may be more flexible for future work.

Let $\nabla, \Delta=\nabla^{\bar{p}} \nabla_{\bar{p}}, R_{\bar{q} p m}^{l}$, etc. and $\hat{\nabla}, \hat{\Delta}, \hat{R}_{\bar{q} p m}{ }^{l}$, etc. be the connections, laplacians, and curvatures with respect to the metrics $g_{\bar{k} j}$ and $\hat{g}_{\bar{k} j}$, respectively. It is most convenient to formulate all the identities we need in terms of the endomorphism $h=h^{\alpha}{ }_{\beta}$ defined by

$$
h_{\beta}^{\alpha}=\hat{g}^{\alpha \bar{\lambda}} g_{\bar{\lambda} \beta}
$$

For example, the difference between the connections and curvatures with respect to $g_{\bar{k} j}$ and $\hat{g}_{\bar{k} j}$ can be expressed as

$$
\begin{aligned}
\nabla_{m} V_{l}-\hat{\nabla}_{m} V_{l} & =-V_{\alpha}\left(\nabla_{m} h h^{-1}\right)^{\alpha}{ }_{l}, \\
\nabla_{m} V^{l}-\hat{\nabla}_{m} V^{l} & =\left(\nabla_{m} h h^{-1}\right)_{\alpha}^{l} V^{\alpha} \\
\hat{R}_{\bar{k} j}{ }^{\alpha}{ }_{\beta}-R_{\bar{k} j}{ }^{\alpha}{ }_{\beta} & =\partial_{\bar{k}}\left(\nabla_{j} h h^{-1}\right)^{\alpha}{ }_{\beta}
\end{aligned}
$$

In particular, taking $V_{l} \rightarrow g_{\bar{k} l}$, we find

$$
\phi_{j \bar{k} m} \equiv \hat{\nabla}_{m} \partial_{\bar{k}} \partial_{j} \phi=g_{\bar{k} \alpha}\left(\nabla_{m} h h^{-1}\right)^{\alpha}{ }_{j}
$$


Henceforth, all indices are raised and lowered with respect to the metric $g_{\bar{k}}$, unless indicated explicitly otherwise. We also set

$$
G=\log \frac{\omega_{\phi}^{n}}{\omega_{0}^{n}} .
$$

Proof of the $C^{2}$ estimates. The basic identity for this step is the following,

$$
\begin{aligned}
\left(\Delta-\partial_{t}\right) \log \operatorname{Tr} h= & \frac{1}{\operatorname{Tr} h}\{\hat{\Delta}(G-\dot{\phi})-\hat{R}\}+\frac{1}{\operatorname{Tr} h} g^{p \bar{q}} g_{\bar{m} j} \hat{g}^{r \bar{m}} \hat{R}_{\bar{q} p}{ }^{j} r \\
& +\left\{\frac{\hat{g}^{\delta \bar{k}} \phi_{\delta \bar{\gamma} p} \phi_{\bar{k}} \bar{\gamma} p}{\operatorname{Tr} h}-\frac{g^{\delta \bar{k}} \partial_{\bar{k}} \operatorname{Tr} h \partial_{\delta} \operatorname{Tr} h}{(\operatorname{Tr} h)^{2}}\right\}
\end{aligned}
$$

This identity follows from another well-known identity [29], which will also be of later use,

$$
\Delta \operatorname{Tr} h=\hat{\Delta} G-\hat{R}+\hat{g}^{\delta \bar{k}} \phi_{\gamma \bar{k} p} \phi^{\gamma} \delta^{p}+g^{p \bar{q}} g_{\bar{m} j} \hat{g}^{r \bar{m}} \hat{R}_{\bar{q} p}{ }^{j}
$$

and can be seen as follows: $\Delta \operatorname{Tr} h=\bar{\Delta} \operatorname{Tr} h=g^{p \bar{q}} \nabla_{\bar{q}} \operatorname{Tr}\left\{\left(\nabla_{p} h h^{-1}\right) h\right\}$, and thus

$$
\Delta \operatorname{Tr} h=g^{p \bar{q}} \operatorname{Tr}\left\{\nabla_{\bar{q}}\left(\nabla_{p} h h^{-1}\right) h\right\}+g^{p \bar{q}} \operatorname{Tr}\left\{\left(\nabla_{p} h h^{-1}\right) \nabla_{\bar{q}} h\right\}
$$

The second term on the right hand side can be recognized as $\hat{g}^{\delta \bar{k}} \phi_{\gamma \bar{k} p} \phi^{\gamma} \delta^{p}$ using (2.25), while, using (2.24), the first term can be rewritten as

$$
\begin{aligned}
g^{p \bar{q}} \operatorname{Tr}\left\{\nabla_{\bar{q}}\left(\nabla_{p} h h^{-1}\right) h\right\} & =g^{p \bar{q}} \hat{R}_{\bar{q} p}{ }^{\alpha}{ }_{\beta} h^{\beta}{ }_{\alpha}-R^{\alpha}{ }_{\beta} h^{\beta}{ }_{\alpha} \\
& =g^{p \bar{q}} g_{\bar{\lambda} \alpha} \hat{R}_{\bar{q} p}{ }^{\alpha}{ }_{\beta} \hat{g}^{\beta \bar{\lambda}}-R^{\alpha}{ }_{\beta} h^{\beta}{ }_{\alpha} .
\end{aligned}
$$

But the Ricci curvature $R_{\bar{\gamma} \beta}$ can be expressed in terms of $G, R_{\bar{\gamma} \beta}=\hat{R}_{\bar{\gamma} \beta}-$ $\partial_{\beta} \partial_{\bar{\gamma}} G$. Substituting in gives (2.28). Taking the log and subtracting the simple identity $\partial_{t} \log \operatorname{Tr} h=(\hat{\Delta} \dot{\phi})(\operatorname{Tr} h)^{-1}$ gives $(2.27)$.

So far the discussion has been general. Let now $\phi$ evolve by the KählerRicci flow,

$$
\dot{\phi}-G=\mu \phi-\hat{f}
$$

so that the term $\hat{\Delta}(\dot{\phi}-G)$ in $(2.27)$ can be replaced by the more tractable term $\mu \hat{\Delta} \phi-\hat{\Delta} \hat{f}$. In [29], it was shown that the expression in brackets 
in (2.27) was always non-negative, while the curvature tensor term was bounded by

$$
\begin{aligned}
-g^{p \bar{q}} g_{m \bar{j}} \hat{R}_{p \bar{q} \alpha}^{j} \hat{g}^{\alpha \bar{m}} & =-\sum_{i, j=1}^{n} \frac{1+\phi_{\bar{i} i}}{1+\phi_{\bar{j} j}} \hat{R}_{\bar{i} i \bar{j} j} \\
& \geq-C(\operatorname{Tr} h) \sum_{j} \frac{1}{1+\phi_{\bar{j} j}},
\end{aligned}
$$

in a system of local holomorphic coordinates where both $g_{\bar{k} j}$ and $\hat{g}_{\bar{k} j}$ were diagonal, and $\hat{g}_{\bar{k} j}$ was the identity matrix at a given point. Thus we have

$$
\begin{aligned}
\left(\Delta-\partial_{t}\right) \log \operatorname{Tr} h & \geq-\mu-C_{1} \frac{1}{\operatorname{Tr} h}-C_{2} \sum_{j=1}^{n} \frac{1}{1+\phi_{\bar{j} j}} \\
& \geq-\mu-C_{3} \sum_{j=1}^{n} \frac{1}{1+\phi_{\bar{j} j}} .
\end{aligned}
$$

Let $A$ be any constant. Since

$$
\Delta \phi=\sum_{j=1}^{n} \frac{\phi_{\bar{j} j}}{1+\phi_{\bar{j} j}}=n-\sum_{j=1}^{n} \frac{1}{1+\phi_{\bar{j} j}^{-}}
$$

we can write

$$
\left(\Delta-\partial_{t}\right)(\log \operatorname{Tr} h-A \phi) \geq C_{4} \dot{\phi}-C_{5}+C_{6} \sum_{j=1}^{n} \frac{1}{1+\phi_{j j}^{-}}
$$

with $A=C_{4}, C_{5}=\mu+A n$, and $C_{6}=A-C_{3}>0$ for $A$ large enough. In view of Perelman's estimate (2.11), we conclude

$$
\left(\Delta-\partial_{t}\right)(\log \operatorname{Tr} h-A \phi) \geq-C_{7}+C_{6} \sum_{j=1}^{n} \frac{1}{1+\phi_{\bar{j} j}} .
$$

Let now $[0, T]$ be any time interval, and $\left(z_{0}, t_{0}\right)$ a point in $X \times[0, T]$ where the function $\log \operatorname{Tr} h-A \phi$ attains its maximum. If this point is not at time $t=0$, then the left hand side of the above equation is $\leq 0$, and we obtain the estimate

$$
\frac{1}{1+\phi_{\bar{j} j}^{-}} \leq C_{8}, \quad 1 \leq j \leq n
$$


But then, at the point $\left(z_{0}, t_{0}\right)$,

$$
\begin{aligned}
\operatorname{Tr} h & =\operatorname{Tr} h\left(\frac{\operatorname{det} \hat{g}_{\bar{k} j}}{\operatorname{det} g_{\bar{k} j}}\right) \mathrm{e}^{\hat{f}-\phi+\dot{\phi}}=\mathrm{e}^{\hat{f}-\phi+\dot{\phi}} \sum_{i=1}^{n}\left(1+\phi_{\overline{i i}}\right) \prod_{j=1}^{n} \frac{1}{1+\phi_{\bar{j} j}} \\
& =\mathrm{e}^{\hat{f}-\phi+\dot{\phi}} \sum_{i=1}^{n} \prod_{j \neq i} \frac{1}{1+\phi_{\bar{j} j}} \leq C_{9}
\end{aligned}
$$

using the boundedness of $\|\phi\|_{C^{0}}$ and again Perelman's estimate. But now we have

$$
\begin{aligned}
\sup _{X \times[0, T]} \operatorname{Tr} h & \leq \mathrm{e}^{A\|\phi\|_{C^{0}}} \exp (\log \operatorname{Tr} h-A \phi)\left(z_{0}, t_{0}\right) \\
& \leq C_{10} .
\end{aligned}
$$

Since $T$ is arbitrary, this establishes the boundedness of the trace of $\hat{g}_{\bar{k} j}+$ $\partial_{j} \partial_{\bar{k}} \phi$, and since the matrix is positive, of all its entries. The proof of the $C^{2}$ estimate is complete.

Proof of the $C^{3}$ estimates. This step was established in [3] when $c_{1}(X)=0$ or $c_{1}(X)<0$, and in [18] for $c_{1}(X)>0$. We shall give below a simpler proof for all cases with completely explicit formulas.

The main ingredient is a parabolic analogue of the Yau, Aubin, and Calabi identities for the third derivatives of the Monge-Ampère equation. In their case, the Ricci curvature is pre-assigned and hence all its derivatives can be controlled. In the present case, we cannot control as yet the derivatives of the Ricci curvature, and it is crucial that they cancel out in the desired identity. We show this by a completely explicit formula, the main technical innovation being the use of the endomorphism $h^{\alpha}{ }_{\beta}$ instead of the potential $\phi$ itself. The squared terms in the Calabi identity arise naturally as the familiar squared terms in a formula of Bochner-Kodaira type.

Let $S$ be defined as in [29] by

$$
S=g^{j \bar{r}} g^{s \bar{k}} g^{m \bar{t}} \phi_{j \bar{k} m} \phi_{\bar{r} s \bar{t}}
$$

In terms of $h^{\alpha}{ }_{\beta}, S$ is just the square of the norm of the $g_{\bar{k} j}$ connection,

$$
S=g^{m \bar{\gamma}} g_{\bar{\mu} \beta} g^{l \bar{\alpha}}\left(\nabla_{m} h h^{-1}\right)^{\beta}{ }_{l} \overline{\left(\nabla_{\gamma} h h^{-1}\right)_{\alpha}}=\left|\nabla h h^{-1}\right|^{2}
$$


and its Laplacian leads immediately to a formula of Bochner-Kodaira type,

$$
\begin{aligned}
\Delta S= & g^{m \bar{\gamma}} g_{\bar{\mu} \beta} g^{l \bar{\alpha}}\left(\Delta\left(\nabla_{m} h h^{-1}\right)^{\beta}{ }_{l} \overline{\left(\nabla_{\gamma} h h^{-1}\right)^{\mu}{ }_{\alpha}}\right. \\
& \left.+\left(\nabla_{m} h h^{-1}\right)_{l}{ }_{l} \bar{\Delta}\left(\nabla_{\gamma} h h^{-1}\right)^{\mu}\right) \\
& +\left|\bar{\nabla}\left(\nabla h h^{-1}\right)\right|^{2}+\left|\nabla\left(\nabla h h^{-1}\right)\right|^{2}
\end{aligned}
$$

where, more explicitly, $\left|\bar{\nabla}\left(\nabla h h^{-1}\right)\right|^{2}=g^{q \bar{p}} g^{j \bar{m}} g^{\beta \bar{\delta}} g_{\bar{\gamma} \alpha} \nabla_{\bar{p}}\left(\nabla_{j} h h^{-1}\right)^{\alpha}{ }_{\beta} \overline{\nabla_{\bar{q}}\left(\nabla_{m} h\right.}$ $\overline{\left.h^{-1}\right)^{\gamma} \delta}$, etc. The relation between $\bar{\Delta}$ and $\Delta$ follows from commuting the $\nabla_{q}$ and the $\nabla_{\bar{p}}$ derivatives,

$$
\begin{aligned}
\left(\bar{\Delta}\left(\nabla_{j} h h^{-1}\right)\right)^{\gamma}{ }_{\alpha}= & \left(\Delta\left(\nabla_{j} h h^{-1}\right)\right)^{\gamma}{ }_{\alpha}-R_{\mu}^{\gamma}\left(\nabla_{\gamma} h h^{-1}\right)^{\mu}{ }_{\alpha} \\
& +R^{\mu}{ }_{\alpha}\left(\nabla_{j} h h^{-1}\right)^{\gamma}{ }_{\mu}+R^{\mu}{ }_{j}\left(\nabla_{\mu} h h^{-1}\right)^{\gamma}{ }_{\alpha}
\end{aligned}
$$

Thus we have

$$
\begin{aligned}
\Delta S= & g^{m \bar{\gamma}} g_{\bar{\mu} \beta} g^{l \bar{\alpha}}\left(\Delta\left(\nabla_{m} h h^{-1}\right)^{\beta}{ }_{l}\right\rangle \overline{\left(\nabla_{\gamma} h h^{-1}\right)_{\alpha}} \\
& +\left(\nabla_{m} h h^{-1}\right)^{\beta} \overline{\Delta\left(\nabla_{\gamma} h h^{-1}\right)^{\mu}{ }_{\alpha}}+\left|\bar{\nabla}\left(\nabla h h^{-1}\right)\right|^{2} \\
& +\left|\nabla\left(\nabla h h^{-1}\right)\right|^{2}+\left(\nabla_{m} h h^{-1}\right)^{\beta}{ }_{l}\left(g^{m \bar{\gamma}} g_{\bar{\mu} \beta} R^{l \bar{\rho}} \overline{\left(\nabla_{\gamma} h h^{-1}\right)^{\mu}}\right. \\
& \left.-g^{m \bar{\gamma}} R_{\bar{\rho} \beta} g^{l \bar{\alpha}} \overline{\left(\nabla_{\gamma} h h^{-1}\right)_{\alpha}}+R^{m \bar{\rho}} g_{\bar{\mu} \beta} g^{l \bar{\alpha}} \overline{\left(\nabla_{\rho} h h^{-1}\right)^{\mu_{\alpha}}}\right)
\end{aligned}
$$

In the case of the elliptic Monge-Ampère equation, this equation suffices already to establish the desired inequality $\Delta S \geq-C_{1} S-C_{2}$. This is because the Ricci tensor $R_{\bar{\alpha} \beta}$ is known in that case, and the Laplacian of $\nabla h h^{-1}$ can be readily reduced to $\nabla R_{\bar{\alpha} \beta}$, using (2.24) and the Bianchi identity,

$$
\begin{aligned}
\Delta\left(\nabla_{j} h h^{-1}\right)_{m}^{l} & =\nabla^{\bar{p}} \partial_{\bar{p}}\left(\nabla_{j} h h^{-1}\right)=-\nabla^{\bar{p}} R_{\bar{p} j m}{ }^{l}+\nabla^{\bar{p}} \hat{R}_{\bar{p} j m}^{l} \\
& =-\nabla_{j} R_{m}^{l}+\nabla^{\bar{p}} \hat{R}_{\bar{p} j m}{ }^{l}{ }^{2}
\end{aligned}
$$

Since the connection $\nabla^{\bar{p}}$ is manifestly $\mathrm{O}\left(\nabla h h^{-1}\right)=\mathrm{O}(\sqrt{S})$, the desired lower bound follows at once. The full expression for $\Delta S$ in terms of $R_{\bar{\alpha} \beta}$ may also be of interest,

$$
\begin{aligned}
& \Delta S=-g^{m \bar{\gamma}} g_{\bar{\mu} \beta} g^{l \bar{\alpha}}\left(\nabla_{m} R^{\beta}{ }_{l} \overline{\left(\nabla_{\gamma} h h^{-1}\right)_{\alpha}}+\left(\nabla_{m} h h^{-1}\right)^{\beta}{ }_{l} \overline{\nabla_{\gamma} R^{\mu}}\right) \\
& +\left|\bar{\nabla}\left(\nabla h h^{-1}\right)\right|^{2}+\left|\nabla\left(\nabla h h^{-1}\right)\right|^{2} \\
& +\left(\nabla_{m} h h^{-1}\right)^{\beta}{ }_{l}\left(g^{m \bar{\gamma}} g_{\bar{\mu} \beta} R^{l \bar{\rho}} \overline{\left(\nabla_{\gamma} h h^{-1}\right)^{\mu}{ }_{\rho}}\right. \\
& \left.-g^{m \bar{g}} R_{\bar{\rho} \beta} g^{l \bar{\alpha}} \overline{\left(\nabla_{\gamma} h h^{-1}\right)_{\alpha}}+R^{m \bar{\rho}} g_{\bar{\mu} \beta} g^{l \bar{\alpha}} \overline{\left(\nabla_{\rho} h h^{-1}\right)_{\alpha}^{\mu}}\right) \\
& +g^{m \bar{\gamma}} g_{\bar{\mu} \beta} g^{l \bar{\alpha}}\left(\nabla^{\bar{p}} \hat{R}_{\bar{p} m}{ }^{\beta}{ }_{l} \overline{\left(\nabla_{\gamma} h h^{-1}\right)_{\alpha}^{\mu}}+\left(\nabla_{m} h h^{-1}\right)^{\beta}{ }_{l} \overline{\nabla^{\bar{p}} \hat{R}_{\bar{p} \gamma^{\mu}}{ }_{\alpha}}\right) \text {. }
\end{aligned}
$$


In the parabolic case, we do not have control of $R_{\bar{\alpha} \beta}$ and its derivatives, and need to eliminate these terms using the time derivative $\dot{S}$ of $S$. We begin by giving a general formula for $\dot{S}$ in terms of $h^{-1} \dot{h}$, so that it is valid for all evolutions. First, note that the derivatives of the connection are given by

$$
\begin{aligned}
\left(\nabla_{j} h\right)^{\cdot} & =\nabla_{j}\left(h^{-1} \dot{h}\right) h+\left(\nabla_{j} h\right)\left(h^{-1} \dot{h}\right), \\
\left(\nabla_{j} h h^{-1}\right)^{\cdot} & =\nabla_{j}\left(h^{-1} \dot{h}\right) .
\end{aligned}
$$

Indeed, note that $\dot{g}_{\bar{\alpha} \beta}=g_{0 \bar{\alpha} \mu} \dot{h}_{\beta}^{\mu}=g_{\bar{\alpha} \nu}\left(h^{-1} \dot{h}\right)^{\nu}{ }_{\beta}=\left(g h^{-1} \dot{h}\right)_{\bar{\alpha} \beta}$, and $\left(g^{\beta \bar{b}}\right)^{\cdot}=$ $-(\dot{g})^{\beta \bar{b}}=-\left(h^{-1} \dot{h}\right)^{\beta}{ }_{\nu} g^{\nu \bar{b}}=-\left(h^{-1} \dot{h} g^{-1}\right)^{\beta \bar{b}}$. Writing

$$
\left\{\left(\nabla_{j} h\right)\right\}^{p}{ }_{q}=g_{\bar{b} q} g^{p \bar{a}} \partial_{j}\left(g^{\beta \bar{b}} g_{\bar{a} \alpha} h_{\beta}^{\alpha}\right)=\left\{g^{-1} \partial_{j}\left(g h g^{-1}\right) g\right\}^{p}{ }_{q}
$$

differentiating with respect to time, and substituting in the preceding formulas for $\dot{g}_{\bar{\alpha} \beta}$ and $\left(\bar{g}^{\bar{\alpha} \beta}\right) \dot{\text { gives at once }}\left(\nabla_{j} h\right)=-h^{-1} \dot{h} \nabla_{j} h+\left(\nabla_{j} h\right)\left(h^{-1} \dot{h}\right)+$ $\nabla_{j}\left(h^{-1} \dot{h} h\right)$, from which the desired formula for $\left(\nabla_{j} h\right)$ follows. The formula for $\left(\nabla_{j} h h^{-1}\right)^{\circ}$ is a simple consequence of the one for $\left(\nabla_{j} h\right)^{*}$. Next, differentiating $S$ gives

$$
\begin{aligned}
\dot{S}= & +g^{m \bar{\gamma}} g_{\bar{\mu} \beta} g^{l \bar{\alpha}}\left(\partial_{t}\left(\left(\nabla_{m} h h^{-1}\right)^{\beta} l\right) \overline{\left(\nabla_{\gamma} h h^{-1}\right)^{\mu}{ }_{\alpha}}\right. \\
& \left.\left.+\left(\nabla_{m} h h^{-1}\right)^{\beta} l\right) \overline{\partial_{t}\left(\nabla_{\gamma} h h^{-1}\right)^{\mu}{ }_{\alpha}}\right) \\
& -\left(\nabla_{m} h h^{-1}\right)^{\beta}{ }_{l}\left(\left(h^{-1} \dot{h}\right)^{m \bar{\gamma}} g_{\bar{\mu} \beta} g^{l \bar{\alpha}} \overline{\left(\nabla_{\gamma} h h^{-1}\right)^{\mu}{ }_{\alpha}}\right. \\
& \left.+g^{m \bar{\gamma}}\left(h^{-1} \dot{h}\right)_{\bar{\mu} \beta} g^{l \bar{\alpha}} \overline{\left(\nabla_{\gamma} h h^{-1}\right)^{\mu}{ }_{\alpha}}-g^{m \bar{\gamma}} g_{\bar{\mu} \beta}\left(h^{-1} \dot{h}\right)^{l \bar{\alpha}} \overline{\left(\nabla_{\gamma} h h^{-1}\right)^{\mu}{ }_{\alpha}}\right)
\end{aligned}
$$

Combining with (2.43), we obtain the following general heat equation,

$$
\begin{aligned}
\left(\Delta-\partial_{t}\right) S= & \left|\bar{\nabla}\left(\nabla h h^{-1}\right)\right|^{2}+\left|\nabla\left(\nabla h h^{-1}\right)\right|^{2} \\
& +g^{m \bar{\gamma}} g_{\bar{\mu} \beta} g^{l \bar{\alpha}}\left\{\left(\Delta-\partial_{t}\right)\left(\nabla_{m} h h^{-1}\right)^{\beta}{ }_{l} \overline{\left(\nabla_{\gamma} h h^{-1}\right)^{\mu}{ }_{\alpha}}\right. \\
& +g^{m \bar{\gamma}} g_{\bar{\mu} \beta} g^{l \bar{\alpha}}\left\{\left(\nabla_{m} h h^{-1}\right)^{\beta} \overline{\left(\Delta-\partial_{t}\right)\left(\nabla_{\gamma} h h^{-1}\right)_{\alpha}}\right. \\
& +\left\{\left(h^{-1} \dot{h}+R\right)^{m \bar{\gamma}} g_{\bar{\mu} \beta} g^{l \bar{\gamma}}-g^{m \bar{\gamma}}\left(h^{-1} \dot{h}+R\right)_{\bar{\mu} \beta} g^{l \bar{\alpha}}\right. \\
& \left.+g^{m \bar{\gamma}} g_{\bar{\mu} \beta}\left(h^{-1} \dot{h}+R\right)^{l \bar{\alpha}}\right\} \times\left(\nabla_{m} h h^{-1}\right)^{\beta}{ }_{l}\left(\nabla_{\gamma} h h^{-1}\right)_{\alpha}^{\mu}
\end{aligned}
$$

We can now specialize to the Kähler-Ricci flow, where

$$
\left(h^{-1} \dot{h}\right)_{l}^{\beta}=-\left(R^{\beta}{ }_{l}-\mu \delta^{\beta}{ }_{l}\right),
$$


and hence, using again (2.24),

$$
\begin{aligned}
\left(h^{-1} \dot{h}+R\right)_{\lambda}^{\beta} & =\mu \delta_{\lambda}^{\beta} \\
\left(\Delta-\partial_{t}\right)\left(\nabla_{j} h h^{-1}\right)_{m}^{l} & =\nabla^{\bar{q}} \hat{R}_{\bar{q} j}^{l}{ }_{m} .
\end{aligned}
$$

Substituting this in the previous formula for $\left(\Delta-\partial_{t}\right) S$, we obtain the following simple and completely explicit parabolic analogue of the $C^{3}$ identity of Yau, Aubin, and Calabi,

$$
\begin{aligned}
\left(\Delta-\partial_{t}\right) S= & \left|\bar{\nabla}\left(\nabla h h^{-1}\right)\right|^{2}+\left|\nabla\left(\nabla h h^{-1}\right)\right|^{2}+\mu\left|\nabla h h^{-1}\right|^{2} \\
& +g^{m \bar{\gamma}} \nabla^{\bar{q}} \hat{R}_{\bar{q} m}{ }^{\beta}{ }_{l} \overline{\left(\nabla_{\gamma} h h^{-1}\right)_{\bar{\beta}} \bar{l}}+g^{m \bar{\gamma}}\left(\nabla_{m} h h^{-1}\right)_{\bar{\mu}}{ }^{\bar{\alpha}} \overline{\nabla \bar{q}} \hat{R}_{\bar{q} \gamma^{\mu}}
\end{aligned}
$$

Note that the terms in $R_{\bar{k} j}$ and its derivatives have cancelled out. Since $\hat{R}_{\bar{q} m}{ }^{l} \beta$ is a fixed tensor, we obtain immediately the estimate

$$
\left(\Delta-\partial_{t}\right) S \geq\left|\bar{\nabla}\left(\nabla h h^{-1}\right)\right|^{2}+\left|\nabla\left(\nabla h h^{-1}\right)\right|^{2}-C_{1} S-C_{2}
$$

The $C^{3}$ estimates for $\phi$ can now be established by the standard arguments: by the $C^{2}$ estimates, the metric $g_{\bar{k} j}$ is known to be equivalent to $\hat{g}_{\bar{k} j}$. Thus $S$ is of the same size as the expression $\hat{g}^{\delta \bar{k}} \phi_{\gamma \bar{k} p} \phi^{\gamma} \delta^{p}$. In view of the identity (2.28), we have for $A$ sufficiently large,

$$
\left(\Delta-\partial_{t}\right)(S+A \hat{\Delta} \phi) \geq C_{3} S-C_{4}
$$

with $C_{3}>0$. The maximum principle implies now that $S$ is bounded by a fixed positive constant. This completes the proof of the $C^{3}$ estimates.

The remaining part of the proof of Lemma 2.4 is standard: the uniform $C^{0}$ and $C^{2}$ estimates for $\phi$ imply uniform $C^{1}$ estimates, and together with the uniform $C^{3}$ estimates, we can deduce that the flow (2.2) is a parabolic equation with uniform $C^{1}$ coefficients. The general theory of parabolic PDE's can then be applied to give uniform $C^{k}$ estimates for all orders $k$.

Proof of Theorem 2.1. Part (i). By Lemmas 2 and 3, the uniform estimate (2.4) implies uniform estimates for $\|\phi\|_{C^{m}}$ for each $m \in \mathbf{N}$. The ArzelaAscoli theorem implies the existence of times $t_{i} \rightarrow+\infty$ with $\phi\left(t_{i}\right)$ and $g_{\bar{k} j}\left(t_{i}\right)$ converging in $C^{\infty}$.

To show that the limit of the subsequence $g_{\bar{k} j}\left(t_{i}\right)$ is a Kähler-Einstein metric, we observe that the uniform boundedness of $\|\phi\|_{C^{m}}$ for each $m \in$ $\mathbf{N}$ implies that the metrics $g_{\bar{k} j}$ are all equivalent along the Kähler-Ricci flow, and that their curvature tensors and their derivatives are all uniformly 
bounded. This is easily seen to imply that the Mabuchi $K$-energy $\nu_{\omega_{0}}(\phi)$ is bounded along the flow, since $\nu_{\omega_{0}}(\phi)$ can be written explicitly as

$$
\begin{aligned}
\nu_{\omega_{0}}(\phi)= & \frac{1}{V} \int_{X}\left\{\log \left(\frac{\omega_{\phi}^{n}}{\omega_{0}^{n}}\right) \omega_{\phi}^{n}-\phi\left(\operatorname{Ric}\left(\omega_{0}\right) \sum_{i=0}^{n-1} \omega_{0}^{i} \omega_{\phi}^{n-1-i}\right.\right. \\
& \left.\left.-\frac{n}{n+1} \sum_{i=0}^{n} \omega_{0}^{i} \omega_{\phi}^{n-i}\right)\right\} .
\end{aligned}
$$

(see also $[4,8]$ for alternative expressions). Next, the lower bound for the Mabuchi energy and the uniform boundedness of the curvature tensor together imply ([21], Theorem 1)

$$
\left\|R_{\bar{k} j}-g_{\bar{k} j}\right\|_{L^{2}} \rightarrow 0, \quad t \rightarrow+\infty
$$

where the $L^{2}$-norm is with respect to $g_{\bar{k} j}$. Since $g_{\bar{k} j}$ is uniformly equivalent to $\hat{g}_{\bar{k} j}$, this holds also for the $L^{2}$ norm with respect to $\hat{g}_{\bar{k} j}$. Returning to the subsequence $g_{\bar{k} j}\left(t_{i}\right)$ converging in $C^{\infty}$, the limit is then a smooth metric $g_{\bar{k} j}^{\infty}$ with $R_{\bar{k} j}^{\infty}-g_{\bar{k} j}^{\infty}=0$, as was to be proved.

To show the full convergence of the Kähler-Ricci flow when $X$ does not admit non-trivial holomorphic vector fields, we show first that, under the assumption of uniform bounds for $\|\phi\|_{C^{m}}$ (and hence for $\left\|g_{\bar{k}_{j}}\right\|_{C^{m}}$ ) for each $m \in \mathbf{N}$, the functions $\dot{\phi}$ converge in $C^{\infty}$. We already know that $\partial_{j} \partial_{\bar{k}} \dot{\phi}=$ $-\left(R_{\bar{k} j}-g_{\bar{k} j}\right) \rightarrow 0$ in all Sobolev norms if the curvature is bounded and the Mabuchi energy is bounded from below ([21], Theorem 1). Since the metrics $g_{\bar{k} j}$, are all equivalent, and all their derivatives are bounded, it follows that $\partial \bar{\partial} \dot{\phi} \rightarrow 0$ in $C^{\infty}$. Next, from the lower boundedness of the Mabuchi energy and the proof of Lemma 2.2, the averages $\alpha(t)=(1 / V) \int_{X} \dot{\phi} \omega_{\phi}^{n}$ of $\dot{\phi}$ converge to 0 as $t \rightarrow \infty$. To deduce the convergence of $\dot{\phi}$ from the convergence of its averages, we write for any constants $\delta, A$,

$$
\begin{aligned}
\dot{\phi}(z)= & \frac{1}{V} \int_{X}(G(z, w)+A)(-\Delta \dot{\phi}(w)+\delta) \omega_{\phi}^{n} \\
& -\delta A+\frac{1}{V} \int_{X} \dot{\phi} \omega_{\phi}^{n}
\end{aligned}
$$

where $G(z, w)$ is the Green's function with respect to $g_{\bar{k} j}$. Since $R_{\bar{k} j} \rightarrow g_{\bar{k} j}$ in $C^{\infty}$, by a theorem of Cheng-Li [6], it follows that we can choose a fixed $A>0$ so that $G(z, w)+A>0$ for all $t$. For any $\epsilon>0$, we can choose $T$ large enough so that $0 \leq(1 / V) \int_{X} \dot{\phi} \omega_{\phi}^{n}<(\epsilon / 2)$ and $-\Delta \dot{\phi}+(\epsilon / 2 A)>0$ for 
all $t>T$. It follows from the preceding identity with $\delta=\epsilon / 2 A$ that

$$
\inf _{X} \dot{\phi} \geq-\frac{\epsilon}{2}, \quad t>T
$$

The same argument with $\dot{\phi}$ replaced by $-\dot{\phi}$ gives the bound $\sup _{X} \dot{\phi} \leq \epsilon$, and thus we have shown that $\dot{\phi} \rightarrow 0$ in $C^{0}$. Together with the convergence of $\partial \bar{\partial} \dot{\phi}$ to 0 in $C^{\infty}$, this implies that $\dot{\phi} \rightarrow 0$ in $C^{\infty}$.

Next, note that the operator $\Delta(t)+1$ is uniformly bounded away from 0 , where $\Delta(t)=\nabla^{\bar{p}} \nabla_{\bar{p}}$ is the scalar Laplacian defined by the evolving metric $g_{\bar{k} j}(t)$. Indeed, assume otherwise. Then there exists a subsequence $t_{i} \rightarrow+\infty$ with Laplacians $\Delta\left(t_{i}\right)+1$ admitting eigenvalues $\lambda\left(t_{i}\right) \rightarrow 0$. By going to a subsequence, we may assume that the corresponding metrics $g_{\bar{k} j}\left(t_{i}\right)$ converge in $C^{\infty}$ to a metric $g_{\bar{k} j}^{\infty}$ which is Kähler-Einstein, by the preceding discussion. The Laplacian $\Delta(\infty)+1$ admits then a zero eigenvalue. The corresponding eigenfunction $u(z)$ defines then a non-trivial holomorphic vector field $V^{j}=$ $g^{\infty j \bar{k}} \partial_{\bar{k}} u$, which contradicts our assumption.

Fix now $t$, and consider the equation in $\psi$

$$
\log \frac{\left(\omega_{\phi(t)}+(i / 2) \partial \bar{\partial} \psi\right)^{n}}{\omega_{\phi(t)}^{n}}+\psi=h
$$

The linearization of the left-hand side at $\psi=0$ is $\Delta(t)+1$. Since this operator is invertible, with uniform bounds in $t$, and since the higher order derivatives of the left-hand side, viewed as a functional in $\psi$, are also uniformly bounded, it follows that there exist constants $\epsilon_{m}>0, A_{m}<\infty$, independent of $t$, so that

$$
\|\psi\|_{C^{m+1}} \leq A_{m}\|h\|_{C^{m}} \text {, when }\|h\|_{C^{m}}<\epsilon_{m}
$$

Finally, let $t, s>>1$. Then the function $\psi \equiv \phi(s)-\phi(t)$ satisfies the equation (2.58), with right hand side $h$ given by

$$
h=\dot{\phi}(t)-\dot{\phi}(s)
$$

For any $\epsilon>0$, we have $\|\dot{\phi}(t)-\dot{\phi}(s)\|_{C^{m}}<\epsilon$ for $s, t$ large enough since $\dot{\phi} \rightarrow 0$ in $C^{m}$. It follows from (2.59) that $\|\phi(s)-\phi(t)\|_{C^{m+1}} \leq A_{m} \epsilon$. This establishes the convergence of the Kähler-Ricci flow (2.2), and hence also of (2.1).

Part (ii). We follow here closely the arguments of Nadel [15] and Demailly-Kollár [7]. If $X$ does not admit a Kähler-Einstein metric, then by part (i) of the theorem, for any $p>1$, there must exist a sequence $\phi\left(t_{i}\right)$ in 
the Kähler-Ricci flow with

$$
\lim _{i \rightarrow \infty} \int_{X} \mathrm{e}^{-p \phi\left(t_{i}\right)} \omega_{0}^{n}=\infty .
$$

Let $\psi$ be an $L^{1}$ limit point of the sequence $\phi\left(t_{i}\right)-\frac{1}{V} \int_{X} \phi\left(t_{i}\right) \omega_{0}^{n}$. Then by the Demailly-Kollár theorem on the semi-continuity of complex singularity exponents ([7], Main Theorem), $\left\|\mathrm{e}^{-\psi}\right\|_{L^{p}(X)}=+\infty$, and hence the multiplier ideal sheaf $\mathcal{J}(p \psi)$ is non-trivial. Equivalently, the corresponding subscheme of structure sheaf $\mathcal{O}_{X} / \mathcal{J}(p \psi)$ is non-empty. Since $p \psi$ is strictly plurisubharmonic with respect to $([p]+1) \omega_{0}$, by the theorem of Nadel [15], in the formulation of Demailly and Kollár ([7], Theorem 4.1 and Corollary 6.6), the multiplier ideal sheaf $\mathcal{J}(p \psi)$ is a coherent analytic sheaf in $\mathcal{O}_{X}$ with $K_{X}^{-[p]} \otimes \mathcal{J}(p \psi)$ having acyclic cohomology, with the $G$-invariance property stated if the metric $\hat{g}_{\bar{k} j}$ is $G$-symmetric.

\section{Remarks}

We conclude with a few simple remarks.

- The estimate (2.20) implies that the functionals $F_{\omega_{0}}(\phi)$ and $F_{\omega_{0}}^{0}(\phi)$ defined by

$$
\begin{aligned}
& F_{\omega_{0}}(\phi)=F_{\omega_{0}}^{0}(\phi)-\log \left(\frac{1}{V} \int_{X} \mathrm{e}^{\hat{f}-\phi} \omega_{0}^{n}\right) \\
& F_{\omega_{0}}^{0}(\phi)=J_{\omega_{0}}(\phi)-\frac{1}{V} \int_{X} \phi \omega_{0}^{n}
\end{aligned}
$$

where $J_{\omega_{0}}(\phi)=1 / 2 V \int_{X} \phi\left(\omega_{0}^{n}-\omega_{\phi}^{n}\right)$ is the Aubin-Yau functional, are bounded by one another along the Kähler-Ricci flow, up to additive constants,

$$
-C_{3}+F_{\omega_{0}}^{0}(\phi) \leq F_{\omega_{0}}(\phi) \leq C_{4}+F_{\omega_{0}}^{0}(\phi)
$$

This may be of some practical use, since while the functional $F_{\omega_{0}}(\phi)$ decreases along the Kähler-Ricci flow, it is the functional $F_{\omega_{0}}^{0}(\phi)$ which is more directly linked to Chow-Mumford stability (see e.g., $[17,20,30])$.

- Since the functional $F_{\omega_{0}}(\phi)$ decreases along the Kähler-Ricci flow, $F_{\omega_{0}}(\phi)$ and hence $F_{\omega_{0}}^{0}(\phi)$ are bounded from above. Thus, along the 
Kähler-Ricci flow, we have

$$
J_{\omega_{0}}(\phi) \leq \frac{1}{V} \int_{X} \phi \omega_{0}^{n}+C .
$$

- The Harnack inequality along the Kähler-Ricci flow proved by TianZhu [26] is the following,

$$
\operatorname{osc}_{X} \phi \leq C_{5} J_{\omega_{0}}(\phi)^{n+\delta}+C_{6}
$$

where $\delta$ is any positive number less or equal to 1 . As shown in [26], Perelman's bound for $\|\dot{\phi}\|_{C^{0}}$ allows arguments similar to the proof of the Harnack inequality for the Monge-Ampère equation $\omega_{\phi}^{n}=\mathrm{e}^{F-t \phi} \omega_{0}^{n}$ $[23,25]$ to take over and establish (3.4).

- The $C^{0}$ estimate $\|\phi\|_{C^{0}} \leq C$ along the Kähler-Ricci flow is now seen to be equivalent to the following estimate on the averages of $\phi$,

$$
\frac{1}{V} \int_{X} \phi \omega_{0}^{n} \leq C .
$$

Indeed, this inequality together with the inequalities (3.3) and (3.4) imply that $\operatorname{osc}_{X} \phi$ is uniformly bounded, which implies in turn that $\|\phi\|_{C^{0}}$ is uniformly bounded, in view of the crude bounds (2.21) for $\sup _{X} \phi$ and $\inf _{X} \phi$. The condition $\omega_{\phi}^{n} \geq C \omega_{0}^{n}$ along the Kähler-Ricci flow introduced by Pali [16] can be interpreted in this light: by Perelman's estimate, this condition is equivalent to $\mathrm{e}^{-\phi} \geq C_{1}>0$, and hence $\sup _{X} \phi \leq C_{2}$, which implies (3.5).

- The application of Kolodziej's theorem to the Kähler-Ricci flow confirms that in his theorem, $p$ cannot be taken to be 1: indeed, $\mathrm{e}^{-\phi}$ is uniformly in $L^{1}(X)$ by the estimate $(2.20)$, so that if the theorem holds for $p=1$, it would follow from the above arguments that the Kähler-Ricci flow always admits a subsequence converging to a Kähler-Einstein metric on a Kähler manifold $X$ with $c_{1}(X)>0$, which is known not to be the case.

- In the applications of multiplier ideal sheaves to the existence of Kähler-Einstein metrics, only the non-existence of non-trivial acyclic multiplier ideal sheaves has played a role so far, and not the value of $p$. It is conceivable that the value of $p$ can carry information. If so, then the multiplier ideal sheaves arising from the Kähler-Ricci flow 
may carry more information than their counterparts from the method of continuity: indeed, as noted above, the condition $p>1$ for the Kähler-Ricci flow is sharp, while this is not known for the condition $(n / n+1)<p<1$ for the method of continuity.

\section{Acknowledgments}

We would like to thank Gabor Szekelyhidi for pointing out to us an oversight in the application of the Nadel vanishing theorem in the original version of this paper. Research supported in part by National Science Foundation grants DMS-02-45371, DMS-06-04657 and DMS-05-14003.

\section{References}

[1] T. Aubin, Metriques riemanniennes et courbure, J. Diff. Geom. 4 (1970), 383-424.

[2] S. Bando and T. Mabuchi, Uniqueness of Einstein-Kähler metrics modulo connected group actions, in 'Algebraic geometry', Sendai, 1985, Advanced Series in Pure Mathematics, Kinokuniya, Tokyo and NorthHolland, Amsterdam, 1987.

[3] H.D. Cao, Deformation of Kähler metrics to Kähler-Einstein metrics on compact Kähler manifolds, Invent. Math. 81 (1985), 359-372.

[4] X.X. Chen, On the lower bound of the Mabuchi energy and its applications, Int. Math. Res. Notices 12 (2000), 607-623.

[5] X.X. Chen and G. Tian, Ricci flow on Kähler-Einstein surfaces, Invent. Math. 147 (2002), 487-544.

[6] S.Y. Cheng and P. Li, Heat kernel estimates and lower bounds of eigenvalues, Comment. Math. Helvetici 56 (1981), 327-338.

[7] J.P. Demailly and J. Kollar, Semi-continuity of complex singularity exponents and Kähler-Einstein metrics on Fano manifolds, Ann. Ec. Norm. Sup 34 (2001), 525-556, arXiv: math.AG/9910118.

[8] W. Ding and G. Tian, Kähler-Einstein metrics and the generalized Futaki invariant, Invent. Math. 110 (1992), 315-335.

[9] S.K. Donaldson, A new proof of a theorem of Narasimhan and Seshadri, J. Diff. Geom. 82 (1983), 279-315. 
[10] S.K. Donaldson, Infinite-dimensional determinants, stable bundles and curvature, Duke Math. J. 54 (1987), 231-247.

[11] J.J. Kohn, Subellipticity for the $\bar{\partial}$-Neumann problem on weakly pseudo-convex domains: sufficient conditions, Acta Math. 142 (1979), $79-122$.

[12] S. Kolodziej, The complex Monge-Ampère equation, Acta Math. 180 (1998), 69-117.

[13] S. Kolodziej, The Monge-Ampère equation on compact Kähler manifolds, Indiana Univ. Math. J. 52 (2003), 667-686.

[14] H. Li, On the lower bound of the $K$ energy and $F$ functional, arXiv: math.DG/0609725.

[15] A. Nadel, Multiplier ideal sheaves and Kähler-Einstein metrics of positive scalar curvature, Ann. of Math. 132 (1990), 549-596.

[16] N. Pali, Characterization of Einstein-Fano manifolds via the KählerRicci flow, arXiv: math.DG /0607581.

[17] S. Paul, Geometric analysis of Chow-Mumford stability, Adv. Math. 182 (2004), 333-356.

[18] G. Perelman, unpublished.

[19] D. Popovici, A Simple Proof of a Theorem by Uhlenbeck and Yau, arXiv: math.CV/0311031.

[20] D.H. Phong and J. Sturm, Stability, energy functionals, and KählerEinstein metrics, Comm. Anal. Geom. 11 (2003), 565-597, MR2015757.

[21] D.H. Phong and J. Sturm, On stability and the convergence of the Kähler-Ricci flow, J. Diff. Geom. 72 (2006), 149-168, arXiv: math.DG/0412185.

[22] N. Sesum and G. Tian, Bounding scalar curvature and diameter along the Kähler Ricci flow (after Perelman) and applications, at www.math.lsa.umich.edu/ lott/ricciflow/perelman.html

[23] Y.T. Siu, The existence of Kähler-Einstein metrics on manifolds with positive anticanonical bundle and a suitable finite symmetry group, Ann. of Math. 127 (1988), 585-627.

[24] Y.T. Siu, Multiplier ideal sheaves in complex and algebraic geometry, arXiv:math.AG/0504259. 
[25] G. Tian, Kähler-Einstein metrics with positive scalar curvature, Invent. Math. 130 (1997), 1-37.

[26] G. Tian and X.H. Zhu, Convergence of the Kähler-Ricci flow, Preprint, 2005.

[27] K. Uhlenbeck and S.T. Yau, The existence of Hermitian Yang-Mills connections on stable bundles over Kähler manifolds, Comm. Pure Appl. Math. 36 (1986), 257-293.

[28] B. Weinkove, A complex Frobenius theorem, multiplier ideal sheaves, and Hermitian-Einstein metrics on stable bundles, Trans. Amer. Math. Soc., to appear, arXiv: math.DG/0306073.

[29] S.T. Yau, On the Ricci curvature of a compact Kähler manifold and the complex Monge-Ampere equation I, Comm. Pure Appl. Math. 31 (1978), 339-411.

[30] S. Zhang, Heights and reductions of semi-stable varieties, Compos. Math. 104 (1996), 77-105.

Department of Mathematics

Columbia University

NEW YORK

NY 10027

USA

E-mail address: phong@cpw.math.columbia.edu

E-mail address: natasas@cpw.math.columbia.edu

Department of Mathematics

Rutgers UniVERSity

NEWARK

NJ 07102

USA

E-mail address: sturm@andromeda.rutgers.edu

Received February 1, 2006 